\title{
C Research Square \\ Contrasting Effects of Plant-Soil Feedbacks on Growth and Morphology of Two Clonal Plants
}

\section{Wei Xue}

Taizhou University https://orcid.org/0000-0002-2230-8570

\section{Lin Huang}

Taizhou University

\section{Wei-Jia Sheng}

Taizhou University

Jia-Tao Zhu

Taizhou University

\section{Shu-Qi Li}

Taizhou University

Fei-Hai Yu ( $\square$ feihaiyu@126.com )

Taizhou University

\section{Research Article}

Keywords: Clonal integration, Clonal growth, Internode length, Plasticity, Plant-soil interactions

Posted Date: July 19th, 2021

DOI: https://doi.org/10.21203/rs.3.rs-705516/v1

License: (c) (i) This work is licensed under a Creative Commons Attribution 4.0 International License.

Read Full License 


\section{Abstract}

Aim

Soil abiotic and biotic conditions are often spatially variable, challenging plants with a heterogeneous environment consisting of favorable and unfavorable patches of soil. Many stoloniferous clonal plants can escape from unfavorable patches by elongating stolon internodes, but aggregate in favorable ones through shortening stolon internodes. However, whether these plants can use their stolons to respond to plant-soil feedbacks (PSFs) is largely unknown.

Methods

In the conditioning phase, we grew either Hydrocotyle vulgaris or Glechoma longituba clonal plants separately in mesocosms to condition bulk soil. In the feedback phase, we grew connected mother and daughter ramets of each species in soil inoculated with the unsterilized or sterilized soil conditioned by conspecifics. We grew the plants for 12 weeks and measured the growth of the mother and daughter ramets separately.

Results

The daughter ramets of $\mathrm{H}$. vulgaris produced more biomass but shorter stolon internodes when grown in soil with sterilized inocula than with unsterilized inocula. However, no difference was found between the daughter ramets of $\mathrm{G}$. longituba grown in soil with unsterilized and sterilized inocula. For both species, no significant difference was found between the mother ramet or between the daughter ramets when the mother ramet was grown in soil with sterilized and unsterilized inocula.

\section{Conclusions}

The daughter ramets rather than the mother ramet of $\mathrm{H}$. vulgaris experienced negative biotic PSFs. However, PSF had no effects on the daughter or mother ramet of G. longituba. Moreover, physiological integration or plasticity in stolon internode lengths cannot help $\mathrm{H}$. vulgaris alleviate the negative PSFs.

\section{Introduction}

Plants can change properties of the soil where they grow, and these changes can in turn influence the performance of other conspecific or heterospecific plants either positively or negatively (Bever 1994; Ehrenfeld et al. 2005; van der Putten et al. 2013). These interactions, also known as plant-soil feedbacks, can facilitate subsequent plant performance through soil nutrient accumulation (Berendse 1990; Chapman et al. 2006) and symbiotic mutualist development (Klironomos 2002; van der Putten et al. 2016), or inhibit it through soil nutrient depletion (Berendse 1994) and pathogen accumulation (van der Putten et al. 2016). However, the negative feedbacks are more commonly observed in nature (Kulmatiski et al. 2008). A large number of plants can propagate vegetatively by producing connected genetically identical individuals (ramets) via clonal growth, and these clonal plants are predominant in many local 
communities (Klimes et al. 1997; Song and Dong 2002; Klimešová et al. 2018). As offspring ramets of clonal plants are commonly produced near their mother ramets, plant-soil feedbacks may affect both the performance of mother and offspring ramets (Cartenì et al. 2012; D'Hertefeldt and van der Putten 1998; van der Stoel et al. 2002).

Many clonal plants can produce rhizomes or stolons of strong plasticity in response to environmental variation in terms of their internode length (de Kroon and Hutchings 1995; Xie et al. 2014). Plasticity of stolon and rhizome internode (hereafter also referred to as "internodes" for brevity) length allows clonal plants to have a higher opportunity to encounter resource-rich patches, and consequently a greater ability to adapt to heterogeneous environments (de Kroon and Hutchings 1995; Gao et al. 2012; Roiloa et al. 2014; van Kleunen and Fischer 2001; Xue et al. 2018c; Ye et al. 2006). In general, clonal plants would produce shorter internodes to utilize adequate resources in favorable habitats, while longer ones can help to "escape" from unfavorable patches (Benedek et al. 2017; de Kroon and Schieving 1990; Dong 1993; Si et al. 2020; Oborny and Cain 1997; Ye et al. 2006). This morphological response in clonal plants has been extensively linked to external environmental factors such as nutrient, water and light availabilities (Dong 1993; Hagiwara et al. 2010; Wijesinghe et al. 2001). However, it is still unclear whether plant-soil feedbacks are involved in such growth strategies. For example, if a clonal plant experiences negative plant-soil feedbacks, it may produce longer internodes to facilitate its offspring ramets to escape from the local stressful environment. By contrast, if a clonal plant experiences positive plant-soil feedbacks, it may produce shorter internodes to maximize use of local soil resources.

When different ramets of the same clone are located in patches of contrasting resource quality, ramets growing in resource-rich patches can translocate nutrients, water and carbohydrates to the ramets growing in resource-poor patches via the connection of internodes (Alpert 1991; Herben et al. 1994; Song et al. 2013; Xue et al. 2020; Yu et al. 2004). The ability to translocate resource among interconnected clonal plants, also known as clonal integration, can benefit certain section(s) of clonal plants experiencing various environmental stresses, including submergence (Luo et al. 2014; Xiao et al. 2010), sand burial and wind erosion (Yu et al. 2004, 2008), salinity (Hester et al. 1994; Xiao et al. 2011) and shading (Li et al. 2018; Stuefer et al. 1994; Xu et al. 2010). Similarly, clonal integration may also benefit clonal plants by reducing effects of negative plant-soil feedbacks or by enhancing the effects of positive plant-soil feedbacks (D'Hertefeldt and van der Putten 1998). However, connection between ramets may also increase the risks of spreading harmful soil microbes (e.g. pathogens) rapidly across connected clones (D'Hertefeldt and van der Putten 1998; Vannier et al. 2018), which may suppress the growth of the clonal plants. Therefore, the response of a clonal plant to plant-soil feedbacks may also depend on the positive and negative effects of biotic plant-soil feedbacks of other connected clonal plants via physiological integration.

Here, we conducted an experiment to examine the effects of biotic plant-soil feedbacks in two wellstudied clonal plants, Hydrocotyle vulgaris and Glechoma longituba (Chen et al. 2014; Liu et al. 2017). We first grew either Hydrocotyle vulgaris or Glechoma longituba clonal plants separately in mesocosms for three months to condition bulk soil. In the feedback phase, we grew connected mother and daughter 
ramets of each of the two species in soil inoculated with the unsterilized or sterilized soil conditioned by conspecifics. Specifically, we tested three hypotheses, for both clonal species: (1) daughter or mother ramets grown in bulk soil inoculated with sterilized (conspecific-conditioned) soil will show greater growth compared with those grown in bulk soil inoculated with unsterilized (conspecific-conditioned) soil, due to accumulation of species-specific soil-borne pathogens in the unsterilized soil (i.e. negative conspecific plant-soil feedbacks); (2) daughter or mother ramets grown in bulk soil inoculated with unsterilized soil will grow better when connected to ramets growing in bulk soil inoculated with sterilized than with unsterilized soil, due to clonal integration; (3) daughter or mother ramets grown in bulk soil inoculated with unsterilized soil will show greater internode growth to show escape response from these unsterilized soil patches.

\section{Materials And Methods}

\section{The species}

Hydrocotyle vulgaris L. (Araliaceae) is a perennial clonal herb that commonly occurs in moist habitats such as bogs, valleys, dune grasslands and moorlands (Dong 1995). It can produce creeping stems with many nodes; each node has the potential to develop into a ramet consisting of a single leaf and adventitious roots (Dong et al. 2015). This species exhibits rapid clonal reproduction and high morphological plasticity (Dong et al. 2015; Dong 1995). Glechoma longituba (Nakai) Kupr. (Lamiaceae) is a perennial clonal herb that can produce ramets connected by stolons. Each ramet has two single leaves originating from a stolon node and some adventitious roots (Liao et al. 2003). The plant is widely distributed in grasslands and forests, on roadsides or by creeks (Liao et al. 2003).

\section{Soil conditioning phase}

To create bulk soil, we collected soil from a barren hill in Taizhou, Zhejiang Province, China, air-dried and being sieved (2-cm-mesh) and then homogeneously mixed with river sand at a 1:1 volume ratio. The bulk soil was used to fill eight pots $(1.5 \mathrm{~L})$, each with $3.5 \mathrm{~kg}$, and grown with plants for soil conditioning. Before filling the pots, we placed a piece of non-woven fiber at the bottom of each pot to avoid soil from running out the pots.

On 28 June 2019, we collected ramets of both $H$. vulgaris and G. Longituba in the campus of Taizhou University in Jiaojiang District, Taizhou, Zhejiang Province, China. We planted these ramets individually in cells $(6.5 \mathrm{~cm} \times 6.5 \mathrm{~cm} \times 6.0 \mathrm{~cm})$ on the seedling plates filled with sterilized potting soil (Hebei Dewoduo Fertilizer Co., LTD, Hengshui, China). Sufficient water was supplied daily. Ten days after transplantation, we selected 20 similar-sized ramets of each species and planted them into the pots. The remaining cultivated ramets were allowed to propagate vegetatively until the start of treatments in the feedback phase as described below.

For each species, we planted five ramets in each pot (Fig. 1) and grown for a period of 12 weeks to condition the soil. Each plant species had four replicates, making a total of 8 pots. We replaced dead 
ramets during the first week of the treatment. All pots were watered daily to promote plant growth.

After 12 weeks, plants from each pot were harvested and large roots were removed from the soil by hand before being homogenized. After which, the soil from each pot was divided into two equal parts. One part of the soil was sterilized by autoclaving at $121^{\circ} \mathrm{C}$ for 120 minutes, and the other part was not sterilized. In total, 16 soil samples were created at the end of the conditioning phase $(2$ species $\times 2$ sterilization treatments $\times 4$ replicates).

\section{Feedback phase}

Each of the 16 soil samples from the conditioning phase was homogenized with the sterilized bulk soil (autoclaving at $121^{\circ} \mathrm{C}$ for 120 minutes) at a ratio of $1: 9(\mathrm{~W}: \mathrm{W})$, resulting in eight soil samples inoculated with the sterilized soils conditioned by either of the two plant species and another eight soil samples inoculated with unsterilized soils conditioned by either of the two plant species. Each of these 16 soil samples were treated as experimental "blocks" and further divided to fill two pots $(12 \mathrm{~cm}$ in diameter and $10 \mathrm{~cm}$ in height) and two polyvinyl chloride (PVC) trays $(100 \mathrm{~cm}$ long $\times 15 \mathrm{~cm}$ wide $\times 8 \mathrm{~cm}$ high; Fig. 1). Four treatment combinations for each plant species were created using these filled pots and trays containing soil inoculated with sterilized or unsterilized soil conditioned by conspecifics: 2 pot soil inoculum treatments (sterilized, unsterilized) $\times 2$ tray soil inoculum treatments (sterilized, unsterilized). Each of these soil treatment combinations were replicated four times, with each set of pot and trays containing sterilized or unsterilized soil inoculum originating from the same soil sample obtained from the end of the conditioning phase.

For each of the two clonal plants, we selected 16 similar-sized ramets (mother ramets) that had developed a primary stolon with two nodes (potential offspring ramets), from the cultivated ramets reserved for the feedback phase. Each mother ramet was planted in a pot containing either unsterilized or sterilized soils conditioned by conspecifics (Fig. 1), and its connected potential daughter ramets by the stolon were grown in a tray containing either unsterilized or sterilized soils conditioned by conspecifics (Fig. 1). Any further primary stolons produced by the mother ramets in the pots and secondary stolons (i.e., side-branches developing from the primary stolon) produced by ramets in the trays were not allowed to root and left trailing from the pots and trays. The feedback phase was maintained for 12 weeks in the greenhouse. We replaced dead ramets during the first week of the experiment. During this experiment, the daily mean temperature in the greenhouse was $23^{\circ} \mathrm{C}$. All pots were watered every two days.

At the end of the experiment, we harvested leaves, stolons and roots for mother (in pots) and daughter ramets (in trays) separately. We also counted number of ramets and measured total stolon length.

Internode length was calculated by using total stolon length divided by the number of nodes along the stolons. All plant materials were oven-dried at $70{ }^{\circ} \mathrm{C}$ for at least $48 \mathrm{hr}$ and weighed.

\section{Data analysis}


We used a linear mixed-effects model to analyze each of the measured variables (i.e., total mass, root mass, stolon mass, leaf mass, number of ramets and internode length). In this model, inoculum treatment (inoculated with the unsterilized vs. sterilized conspecific-conditioned soil) of the mother ramets, inoculum treatment of the daughter ramets and their interaction were included as fixed factors. Pot identity in the conditioning phase was included as a random factor in order to account for the nonindependence of the inoculum. We performed separate linear mixed-effects models for measurements of the daughter ramets, the mother ramets and the whole clone (daughter + mother ramets). In these analyses, significant local effects of inoculum treatment of the daughter (or mother) ramets on the growth of the daughter (or mother) ramets indicate significant plant-soil feedback effects; while significant nonlocal effects of inoculum treatment of the daughter (or mother) ramets on the growth of the mother (or daughter) ramets indicate clonal integration effects (Stuefer et al. 1994). When a significant effect was detected, Tukey tests were used for post-hoc comparisons.

All analyses were performed with R (version 3.4.4; http://www.r-project.org) in RStudio (version 1.1.423; http://rstudio.org). Linear mixed-effects models were fitted with the $n / m e$ package (version 3.1-128; Pinheiro et al., 2016); Post-hoc comparisons were made using the glht function in the multcomp package (version 1.4-15). All data were checked graphically for normality and homogeneity of variance. Total mass, root mass, leaf mass and internode length of the whole clone of $H$. vulgaris and total mass of the whole clone of $G$. longituba were log-transformed before analysis.

\section{Results}

\section{Effects on growth and morphology of $H$. vulgaris}

In $\mathrm{H}$. vulgaris, biomass (total mass, root mass, stolon mass and leaf mass) of the daughter ramets was significantly greater when they were grown in trays containing sterilized conspecific-conditioned soil (LS and SS) than when they were grown in trays containing unsterilized conspecific-conditioned soil (LL and SL; Fig. 2A-D; Table 1A), indicating that there was a significant negative plant-soil feedback effect on the growth of the daughter ramets of $\mathrm{H}$. vulgaris. However, the inoculum treatment of the mother ramet did not influence the growth of the daughter ramets, indicating that there was no integration effect on the daughter ramets. Internode length of the daughter ramets was also greater when they were grown in trays containing sterilized conspecific-conditioned soil (LS and SS) than when they were grown in trays containing unsterilized conspecific-conditioned soil (LL and SL; Fig. 2F; Table 1A), indicating that daughter ramets of $H$. vulgaris did not show escape strategy in response to the negative plant-soil feedbacks. There was no treatment effect on the ramet number of the daughter ramets of $H$. vulgaris (Fig. 2E; Table 1A).

The inoculum treatment of the mother ramet or daughter ramets did not influence the growth or morphology of the mother ramet of $\mathrm{H}$. vulgaris (Fig. 2; Table 1B), indicating that there was no significant effect of plant-soil feedback or integration on the mother ramet. 
The growth and morphology of the whole-clone showed a similar pattern as those of its daughter ramets, except for a significant interactive effect on root mass of $H$. vulgaris (Fig. 2; Table 1).

\section{Effects on growth and morphology of G. longituba}

In $G$. longituba, the inoculum treatment of the daughter or mother ramets did not influence the growth measures (total mass, root mass, stolon mass, leaf mass and number of ramtes) of the daughter ramets (Fig. 3A-E; Table 2A), indicating that there was no significant effect of plant-soil feedback or integration on the daughter ramets of $G$. longituba. However, internode length of the daughter ramets of $G$. longituba was overall greater when the mother ramet was grown in pots containing unsterilized conspecificconditioned soil (LL and LS) than when it was grown in pots containing sterilized conspecific-conditioned soil (SL and SS; Fig. 3F; Table 2B), indicating a significant integration effect on the morphology of the daughter ramets.

There was no significant treatment effect on the growth or morphology of the mother ramets of $G$. longituba (Fig. 3; Table 2B).

Total mass, root mass and internode length of the whole clone of $G$. longituba were significantly greater when the mother ramet was grown in pots containing unsterilized conspecific-conditioned soil (LL and LS) than when it was grown in pots containing sterilized conspecific-conditioned soil (SL and SS; Fig. 3A, $B$ and F; Table 2C). Stolon mass and leaf mass of the whole clone of $G$. longituba was significantly greater when the daughter ramets were grown in trays containing sterilized conspecific-conditioned soil (LS and SS) than when they were grown in trays containing unsterilized conspecific-conditioned soil (LL and SL; Fig. 2C-D; Table 2C). Ramet number of the whole clone was not influenced by the inoculum treatments of the mother or daughter ramets (Fig. 2E; Table 2C).

\section{Discussion}

Our results showed that the daughter ramets rather than the mother ramet of $\mathrm{H}$. vulgaris experienced negative biotic plant-soil feedbacks, but no biotic plant-soil feedbacks were found for either the daughter or mother ramets of $G$. longituba. Moreover, there was no evidence of facilitation by the $H$. vulgaris mother ramets grown in sterilized conspecific-conditioned soil

to their connected daughter ramets suffered from negative biotic plant-soil feedbacks. More importantly, the daughter ramets of $\mathrm{H}$. vulgaris did not show "escape" responses in terms of internode length from the negative biotic plant-soil feedbacks. These results indicated that biotic plant-soil feedbacks not only differed among different species, but also differed in physically-connected ramets of the same clone. However, physiological integration and stolon plasticity may not be involved in the response of the two clonal plants to the biotic plant-soil feedbacks as what they usually do in response to abiotic stresses (Gruntman et al. 2017; Roiloa et al. 2010; Yu et al. 2004).

Biotic plant-soil feedbacks vary greatly among different plant species, as well as among different genotypes of the same species (Klironomos 2002; Wagg et al. 2015; Xue et al. 2018a). Here, our results 
further revealed that biotic plant-soil feedbacks may also differ between the connected ramets of the same clone in $\mathrm{H}$. vulgaris. These differences may have important implications for the thriving of the clonal plants in nature as this variation may "blur" the negative feedbacks on the whole clone (Hart et al. 2016).

Although interconnected, the daughter ramets were suppressed by the negative biotic plant-soil feedbacks, but the mother ramet was not. This result was contrast to a previous study showing that soilborne pathogens had greatly influenced the mother ramets of a rhizomatous clonal grass Carex arenaria, but the daughter ramets were optimally defended (D'Hertefeldt and van der Putten 1998). This was explained by the transportation of resources (physiological integration) from the mother ramets to support their connected daughter ramets, as reported in also many other studies (Lu et al. 2020; Song et al. 2013; Wang et al. 2017). In the present study, however, we did not observe a significant facilitation of the mother ramet of $H$. vulgaris to its connected daughter ramets. Therefore, our second hypothesis was not supported. We proposed that local plant defense may have played a major role in driving the distinct responses of the mother and daughter ramets to biotic plant-soil feedbacks (Stuefer et al. 2004). The daughter ramets were more vulnerable in facing species-specific soil pathogens, compared to the mother ramet, despite that they had access to more resources in terms of nutrients and spaces.

While $H$. vulgaris daughter ramets were affected by negative biotic plant-soil feedbacks, it did not produce longer stolon internodes. This result thus does not support our hypothesis that clonal plants can elongate their inter-ramet distance to facilitate their escape from negative plant-soil feedbacks. Studies have shown that clonal plants can elongate their internodes under stressful conditions such as low light intensity (de Kroon and Hutchings 1995), low nutrient availability (Thomas and Hay 2008) and drought (Zhao et al. 2008). However, under stressful conditions, stolon internodes can be shortened rather than elongated compared to non-stressful conditions, as plants may switch their development from vegetative growth to flowering (Navas and Garnier 2002; Stuefer et al. 2004; Ye et al. 2006; Yu and Dong 2003). This is likely the case in our study, as we found that stolon internode of $H$. vulgaris was shortened under the negative effects of plant-soil feedbacks. This result indicated that negative plant-soil feedbacks may less far-reaching than stolon length. Therefore, it may still not be a problem for this species to be flourishing in natural circumstances despite that they cannot escape negative plant-soil feedbacks through elongating stolons, because they may also use the seeds to distribute to better conditions.

We conclude that biotic plant-soil feedbacks can negatively influence the growth of the daughter ramets, rather than the mother ramet, consequently reduce the growth of the whole clone. However, we did not find the evidence that physiological integration can facilitate the growth of the daughter ramets suffered from negative feedbacks, and that the daughter ramets cannot escape from such negative feedbacks through elongation of internode length either. These results were largely attributed to the defenses of the daughter and mother ramets against their local species-specific soil pathogens. However, we should notice that these results were only true for $H$. vulgaris, but not for $G$. longituba, and thus a general conclusion requires investigations with a large number of clonal plants. Moreover, we constrained the growth of the daughter and mother ramets in separate containers where the soil could be treated as 
homogeneous by the daughter and mother ramets. However, soil abiotic and biotic conditions are often spatially variable in natural circumstance, challenging plants with a heterogeneous environment consisting of more and less favorable patches of soil (Xue et al. 2018b). It is still unknown whether clonal plants can show escape strategies in response to negative plant-soil feedbacks in a heterogeneous soil.

\section{Declarations}

\section{Declaration of Competing Interest}

None.

\section{Acknowledgements}

We thank Robin Heinen (Technical University of Munich) and two anonymous reviewers for their constructive comments on an early version of the manuscript. Research was supported by the National Natural Science Foundation of China (grants 32001122 and 31761123001), the Joint Fund of Zhejiang Provincial Natural Science Foundation (grant LTZ20C030001) and the Ten-Thousand-Talent Program of Zhejiang Province (grant 2018R52016).

\section{References}

Alpert P (1991) Nitrogen sharing among ramets increases clonal growth in Fragaria chiloensis. Ecology 72:69-80

Benedek V, Englert P, Oborny B (2017) The effect of branching angle on adaptive growth in patchy environments. Evol Ecol 31:333-344

Berendse F (1990) Organic matter accumulation and nitrogen mineralization during secondary succession in heathland ecosystems. J Ecol 78:413-427

Berendse F (1994) Litter decomposability-a neglected component of plant fitness. J Ecol 82:187-190

Bever JD (1994) Feeback between plants and their soil communities in an old field community. Ecology 75:1965-1977.

Cartenì F, Marasco A, Bonanomi G, Mazzoleni S, Rietkerk M, Giannino F (2012) Negative plant soil feedback explaining ring formation in clonal plants. J Theor Biol 313:153-161

Chapman SK, Langley JA, Hart SC, Koch GW (2006) Plants actively control nitrogen cycling: uncorking the microbial bottleneck. New Phytol 169:27-34

Chen J-S, Li J, Zhang Y, Zong H, Lei N-F (2014) Clonal integration ameliorates the carbon accumulation capacity of a stoloniferous herb, Glechoma longituba, growing in heterogenous light conditions by facilitating nitrogen assimilation in the rhizosphere. Ann Bot, 115: 127-136 
D'Hertefeldt T, van der Putten WH (1998) Physiological integration of the clonal plant Carex arenaria and its response to soil-borne pathogens. Oikos 81:229-237

de Kroon H, Hutchings MJ (1995) Morphological plasticity in clonal plants: the foraging concept reconsidered. J Ecol 83:143-152

de Kroon H, Schieving F (1990) Resource partitioning in relation to clonal growth strategy. Pages 113-130 in Van Groenendael J, de Kroon $\mathrm{H}$, eds. Clonal Growth in Plants: Regulation and Function. The Hague: SPB Academic Publishing

Dong B-C, Wang J-Z, Liu R-H, Zhang M-X, Luo F-L, Yu F-H (2015) Soil heterogeneity affects ramet placement of Hydrocotyle vulgaris. J Plant Ecol 8:91-100

Dong M (1993) Morphological plasticity of the clonal herb Lamiastrum galeobdolon (L.) Ehrend. \& Polatschek in response to partial shading. New Phytol 124:291-300

Dong M (1995) Morphological responses to local light conditions in clonal herbs from contrasting habitats, and their modification due to physiological integration. Oecologia 101:282-288

Ehrenfeld JG, Ravit B, Elgersma K (2005) Feedback in the plant-soil system. Annu Rev Env Resour 30:75115

Gao Y, Xing F, Jin Y, Nie D, Wang Y (2012) Foraging responses of clonal plants to multi-patch environmental heterogeneity: spatial preference and temporal reversibility. Plant Soil 359:137-147

Gruntman M, Groß D, Májeková M, Tielbörger K. (2017) Decision-making in plants under competition. Nat Commun 8:2235

Hagiwara Y, Kachi N, Suzuki J (2010) Effects of temporal heterogeneity of water supply on the growth of Perilla frutescens depend on plant density. Ann Bot 106:173-181

Hart SP, Schreiber SJ, Levine JM (2016) How variation between individuals affects species coexistence. Ecol Lett, 19: 825-838

Hester MW, Mckee KL, Burdick DM, Koch MS (1994) Clonal integration in Spartina patens across a nitrogen and salinity gradient. Can J Bot 72:767-770

Herben T, Hara T, Marshall C, Soukupová L (1994) Plant clonality: Biology and diversity. Folia Geobot 29:113-122

Klimes L, Klimesova J, Hendriks R, van Groenendael J (1997) Clonal plant architecture: a comparative analysis of form and function. Pages 1-29 in de Kroon H, van Groenendael J, eds. The ecology and evolution of clonal plants. Leiden: Backhuys 
Klimešová J, Martínková J, Herben T (2018) Horizontal growth: An overlooked dimension in plant trait space. Perspect Plant Ecol Evol Syst 32:18-21

Klironomos JN (2002) Feedback with soil biota contributes to plant rarity and invasiveness in communities. Nature 417:67-70

Kulmatiski A, Beard KH, Stevens JR, Cobbold SM (2008) Plant-soil feedbacks: a meta-analytical review. Ecol Lett 11:980-992

Li Y, Chen J-S, Xue G, Peng Y, Song H-X (2018) Effect of clonal integration on nitrogen cycling in rhizosphere of rhizomatous clonal plant, Phyllostachys bissetii, under heterogeneous light. Sci Total Environ 628-629:594-602

Liao M, Yu F, Song M, Zhang S, Zhang J, Dong M (2003) Plasticity in R/S ratio, morphology and fitnessrelated traits in response to reciprocal patchiness of light and nutrients in the stoloniferous herb, Glechoma longituba L. Acta Oecol 24:231-239

Liu L, Alpert P, Dong B-C, Li J-M, Yu F-H (2017) Combined effects of soil heterogeneity, herbivory and detritivory on growth of the clonal plant Hydrocotyle vulgaris. Plant Soil, 421: 429-437

Lu H-Z, Brooker R, Song L, Liu W-Y, Sack L, Zhang J-L, Yu F-H (2020) When facilitation meets clonal integration in forest canopies. New Phytol 225:135-142

Luo F-L, Chen Y, Huang L, Wang A, Zhang M-X, Yu F-H (2014) Shifting effects of physiological integration on performance of a clonal plant during submergence and de-submergence. Ann Bot 113:1265-1274

Navas M-L, Garnier E (2002) Plasticity of whole plant and leaf traits in Rubia peregrina in response to light, nutrient and water availability. Acta Oecol 23:375-383

Oborny B, Cain ML (1997) Models of spatial spread and foraging in clonal plants. Pages 155-183 in de Kroon H, van Groenendael J, eds. The ecology and evolution of clonal plants. Leiden: Backbuys Publishers

Pinheiro JC, Bates DM, DebRoy S, Sarkar D, Team RDC (2016) nlme: Linear and nonlinear mixed effects models. $\mathrm{R}$ package version 3.1-128.

Roiloa SR, Rodríguez-Echeverría S, de la Pena E, Freitas H (2010) Physiological integration increases the survival and growth of the clonal invader Carpobrotus edulis. Biol Invas 12:1815-1823

Si C, Xue W, Lin J, Zhang J-F, Hong M-M, Wang Y-Y, Zhang L-F, Yu F-H (2020) No evidence of greater biomass allocation to stolons at moderate resource levels in a floating plant. Aquat Ecol 54:421-429

Song M-H, Dong M (2002) Clonal plants and plant species diversity in wetland ecosystems in China. J Veg Sci 13:237-244 
Song Y-B, Yu F-H, Keser LH, Dawson W, Fischer M, Dong M, van Kleunen M (2013) United we stand, divided we fall: a meta-analysis of experiments on clonal integration and its relationship to invasiveness. Oecologia 171:317-327

Stuefer JF, During HJ, de Kroon H (1994) High benefits of clonal integration in two stoloniferous species, in response to heterogeneous light environments. J Ecol 82:511-518

Stuefer JF, Gomez S, van Molken T (2004) Clonal integration beyond resource sharing: implications for defence signalling and disease transmission in clonal plant networks. Evol Ecol, 18: 647-667

Thomas R, Hay M (2008) Adaptive variation in physiological traits underpinning stem elongation responses among nodally-rooting stoloniferous herbs. Evol Ecol 22:369-381

van der Putten WH, Bardgett RD, Bever JD, Bezemer TM, Casper BB, Fukami T, Kardol P, Klironomos JN, Kulmatiski A, Schweitzer JA, Suding KN, van de Voorde TFJ, Wardle DA (2013) Plant-soil feedbacks: the past, the present and future challenges. J Ecol 101:265-276

van der Putten WH, Bradford MA, Brinkman EP, van der Voorde TFJ, Veen GF (2016) Where, when and how plant-soil feedback matters in a changing world. Funct Ecol 30:1109-1121

van der Stoel CD, van der Putten WH, Duyts H (2002) Development of a negative plant-soil feedback in the expansion zone of the clonal grass Ammophila arenaria following root formation and nematode colonization. J Ecol 90:978-988

van Kleunen M, Fischer M (2001) Adaptive evolution of plastic foraging responses in a clonal plant. Ecology 82:3309-3319

Wagg C, Boller B, Schneider S, Widmer F, van der Heijden MGA (2015) Intraspecific and intergenerational differences in plant-soil feedbacks. Oikos, 124: 994-1004

Wang Y-J, Müller-Schärer H, Kleunen M, Cai A-M, Zhang P, Yan R, Dong B-C, Yu F-H (2017) Invasive alien plants benefit more from clonal integration in heterogeneous environments than natives. New Phytol 216:1072-1078

Wijesinghe DK, John EA, Beurskens S, Hutchings MJ (2001) Root system size and precision in nutrient foraging: responses to spatial pattern of nutrient supply in six herbaceous species. J Ecol 89:972-983

Xiao Y, Tang J, Qing H, Ouyang Y, Zhao Y, Zhou C, An S (2010) Clonal integration enhances flood tolerance of Spartina alterniflora daughter ramets. Aquat Bot 92:9-13

Xiao Y, Tang J, Qing H, Zhou C, An S (2011) Effects of salinity and clonal integration on growth and sexual reproduction of the invasive grass Spartina alterniflora. Flora 206:736-741 
Xie X-F, Song Y-B, Zhang Y-L, Pan X, Dong M (2014) Phylogenetic meta-analysis of the functional traits of clonal plants foraging in changing environments. PLoS ONE 9:e107114

Xu C-Y, Schooler SS, Klinken RDV (2010) Effects of clonal integration and light availability on the growth and physiology of two invasive herbs. J Ecol 98:833-844

Xue W, Bezemer TM, Berendse F (2018a) Density-dependency and plant-soil feedback: former plant abundance influences competitive interactions between two grassland plant species through plant-soil feedbacks. Plant Soil 428:441-452

Xue W, Berendse F, Bezemer TM (2018b) Spatial heterogeneity in plant-soil feedbacks alters competitive interactions between two grassland plant species. Funct Ecol 32:2085-2094

Xue W, Huang L, Yu F-H, Bezemer TM (2018c) Intraspecific aggregation and soil heterogeneity:

competitive interactions of two clonal plants with contrasting spatial architecture. Plant Soil 425:231-240

Xue W, Wang W-L, Yuan Q-Y, Yu F-H (2020) Clonal integration in Phragmites australis alters soil microbial communities in an oil-contaminated wetland. Environ Pollut 265:114828

Ye X-H, Yu F-H, Dong M (2006) A trade-off between guerrilla and phalanx growth forms in Leymus secalinus under different nutrient supplies. Ann Bot 98:187-191

Yu F-H, Wang N, He W-M, Chu Y, Dong M (2008) Adaptation of rhizome connections in drylands: increasing tolerance of clones to wind erosion. Ann Bot 102:571-577

Yu F-H, Dong M (2003) Effect of light intensity and nutrient availability on clonal growth and clonal morphology of the stoloniferous herb Halerpestes ruthenica. J Integr Plant Biol 45:408-416

Yu F-H, Dong M, Krusi B (2004) Clonal integration helps Psammochloa villosa survive sand burial in an inland dune. New Phytol 162:697-704

Zhao W, Chen S-P, Lin G-H (2008) Compensatory growth responses to clipping defoliation in Leymus chinensis (Poaceae) under nutrient addition and water deficiency conditions. Plant Ecol 196:85-99

\section{Tables}

Table 1 Effects of the inoculum treatment (inoculated with the unsterilized vs. sterilized conspecificconditioned soil) of the mother and daughter ramets on the growth and morphology of (A) the daughter ramets, (B) the mother ramet and (C) the whole clone (mother plus daughter ramets) of Hydrocotyle vulgaris 
Effect

\begin{tabular}{|c|c|c|c|c|c|}
\hline Total & Root & Stolon & Leaf & No. of & Internode \\
\hline mass & mass & mass & mass & ramets & length \\
\hline
\end{tabular}

(A) Daughter ramets

\begin{tabular}{llllllll} 
Mother inoculum (M) & 1,9 & 0.03 & 1.35 & 0.12 & 0.51 & 0.20 & 0.71 \\
\hline Daughter inoculum (D) & 1,9 & $17.32^{\star \star}$ & $16.62^{\star \star}$ & $12.97^{\star \star}$ & $11.18^{\star \star}$ & 2.28 & $23.47^{\star \star \star}$ \\
\hline M x D & 1,9 & $<0.01$ & 2.16 & 0.52 & 0.92 & 0.07 & 0.26
\end{tabular}

(B) Mother ramets

\begin{tabular}{llllllll} 
Mother inoculum (M) & 1,9 & 1.49 & 1.83 & 1.09 & 1.04 & 1.04 & 0.77 \\
\hline Daughter inoculum (D) & 1,9 & 1.56 & 2.76 & 1.39 & 0.27 & 0.41 & 1.66 \\
\hline M x D & 1,9 & 2.98 & 6.98 & 1.53 & 2.23 & 0.25 & 0.78
\end{tabular}

(C) Whole clone

\begin{tabular}{llllllll} 
Mother inoculum (M) & 1,9 & 0.22 & 0.59 & 0.21 & 1.06 & 0.59 & 0.44 \\
\hline Daughter inoculum (D) & 1,9 & $21.82^{\star \star}$ & $92.83^{\star \star \star}$ & $14.51^{\star \star}$ & $10.52^{*}$ & 2.01 & $11.97^{\star \star}$ \\
\hline M x D & 1,9 & 0.45 & $33.76^{\star \star \star}$ & 0.08 & 2.20 & 0.39 & 0.03
\end{tabular}

Numbers are $F$-values of two-way ANOVAs. Superscripts give $P$ : ${ }^{* \star} P<0.001,{ }^{* \star} P<0.01$ and ${ }^{*} P<0.05$. Total mass, root mass, leaf mass and internode length of the whole clone were log-transformed.

Table 2 Effects of the inoculum treatment (inoculated with the unsterilized vs. sterilized conspecificconditioned soil) of the mother and daughter ramets on the growth and morphology of (A) the daughter ramets, $(B)$ the mother ramets and $(C)$ the whole clone (mother plus daughter ramets) of Glechoma longituba 
Effect

DF Total Root Stolon Leaf No. of Internode mass mass mass mass ramets length

(A) Daughter ramets

\begin{tabular}{|llllllll}
\hline Mother inoculum (M) & 1,9 & 2.28 & 1.05 & 2.15 & 2.65 & 2.85 & $6.18^{*}$ \\
\hline Daughter inoculum (D) & 1,9 & 3.86 & 3.43 & 2.75 & 3.97 & 1.87 & 1.98 \\
\hline M x D & 1,9 & 1.18 & 0.95 & 1.35 & 0.84 & 0.06 & 0.96
\end{tabular}

(B) Mother ramets

\begin{tabular}{|llllllll} 
Mother inoculum (M) & 1,9 & 0.12 & 0.42 & 0.23 & 0.01 & 0.60 & 0.01 \\
\hline Daughter inoculum (D) & 1,9 & 1.39 & 1.42 & 2.14 & 0.66 & 0.60 & 0.60 \\
\hline M x D & 1,9 & 0.21 & 0.57 & 0.07 & 0.09 & 0.02 & 0.01
\end{tabular}

(C) Whole clone

\begin{tabular}{llllllll} 
Mother inoculum (M) & 1,9 & $1.85^{\star \star}$ & $0.83^{\star \star}$ & 1.99 & 3.99 & 0.66 & $8.14^{\star}$ \\
\hline Daughter inoculum (D) & 1,9 & 14.92 & 12.13 & $6.34^{\star}$ & $7.67^{\star}$ & 3.97 & 3.73 \\
\hline M x D & 1,9 & 2.43 & 3.74 & 1.99 & 1.54 & 0.01 & 1.36
\end{tabular}

Numbers are $F$-values of two-way ANOVAs. Superscripts give $P$ : ${ }^{*} P<0.01$ and ${ }^{\star} P<0.05$. Total mass of the whole clone was log-transformed.

\section{Figures}




\section{Conditioning phase}

\section{Feedback phase}

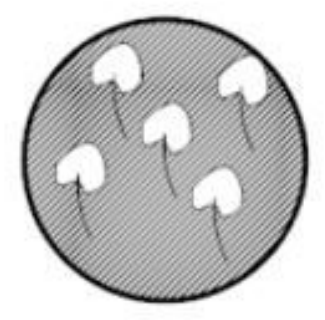

LL

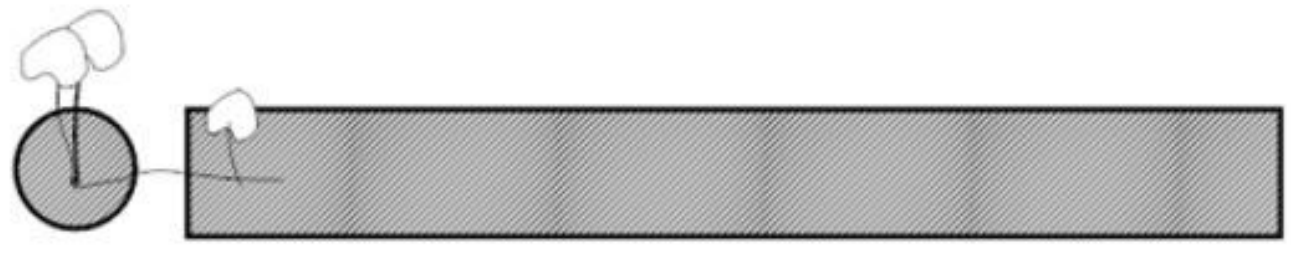

LS

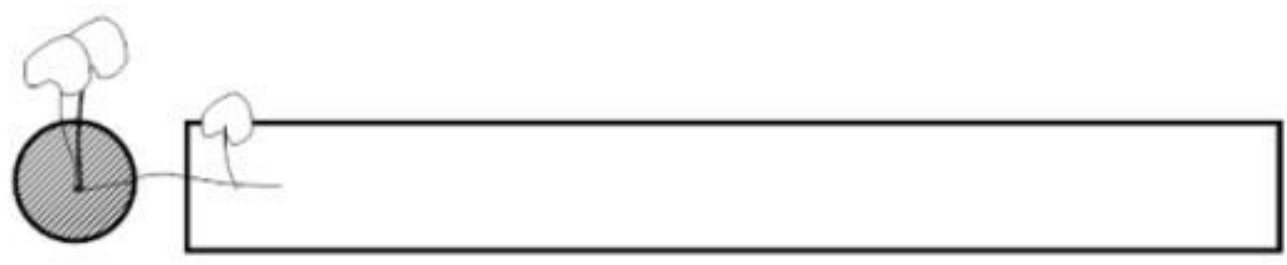

$\mathrm{SL}$

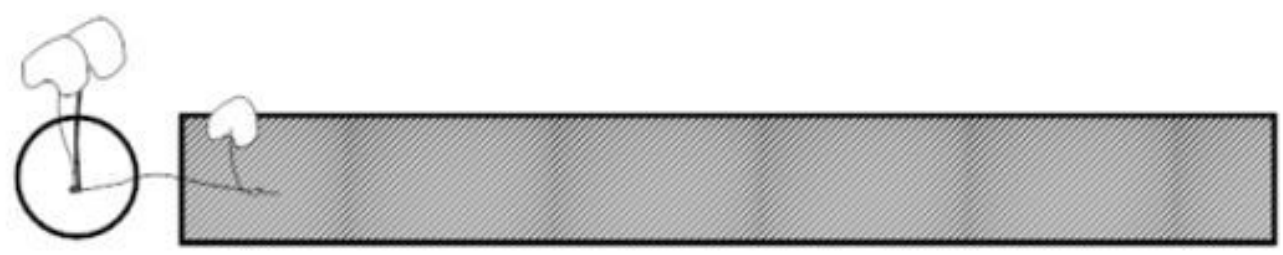

SS

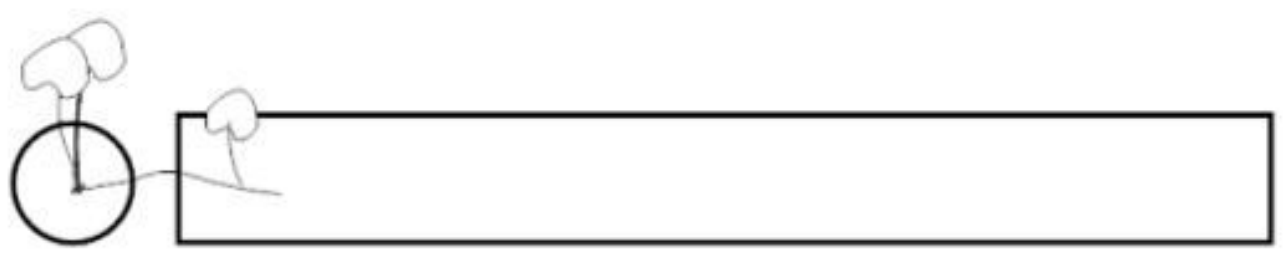

\section{Figure 1}

Schematic representation of the experimental design. The experiment consisted of two phases. In the conditioning phase, the soil in each pot was grown with five ramets of Hydrocotyle vulgaris or Glechoma longituba for 12 weeks. In the feedback phase, for each species, a mother ramet was grown in a pot (circle) filled with a bulk soil inoculated with either a unsterilized or a sterilized conspecific-conditioned soil collected from the conditioning phase, and its daughter ramets connected by a primary stolon were grown in a tray (rectangle) filled with the bulk soil inoculated with either a unsterilized or sterilized conspecific-conditioned soil collected from the conditioning phase. Treatment codes: LL - both mother and daughter ramets were grown in the bulk soil inoculated with the unsterilized conspecific-conditioned 
soil, LS - the mother and daughter ramets were grown in the bulk soil inoculated with the unsterilized and sterilized conspecific-conditioned soil, respectively, SL - the mother and daughter ramets were grown in the bulk soil inoculated with the sterilized and unsterilized conspecific-conditioned soil, respectively, and SS - both mother and daughter ramets were grown in the bulk soil inoculated with the sterilized conspecific-conditioned soil.
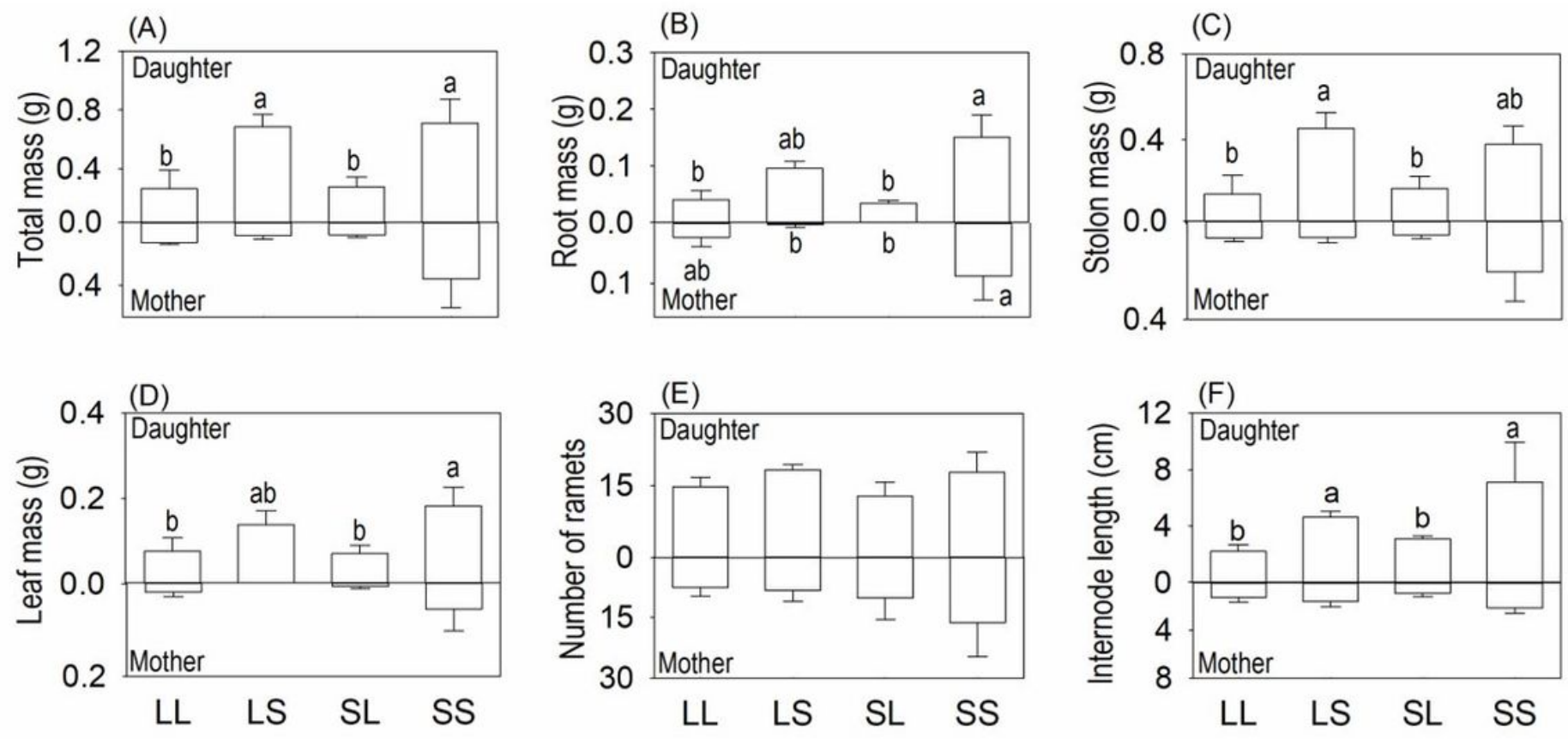

\section{Figure 2}

Total mass (A), root mass (B), stolon mass (C), leaf mass (D), number of ramets (E) and internode length (F) of the daughter (upper panel) and mother (lower panel) ramets of Hydrocotyle vulgaris under the four treatments $(N=4)$. Mean values $(S E)$ are given. Different letters indicate significant difference among the treatments. Conspecific-conditioned soil inoculation treatments: LL - "mother" and "daughter" ramets grown in soil inoculated with unsterilized conspecific soil, LS - mother with unsterilized and daughter with sterilised inoculum, SL - mother with sterilized and daughter with unsterilized inoculum, and SS - mother and daughter with sterilized inoculum. 

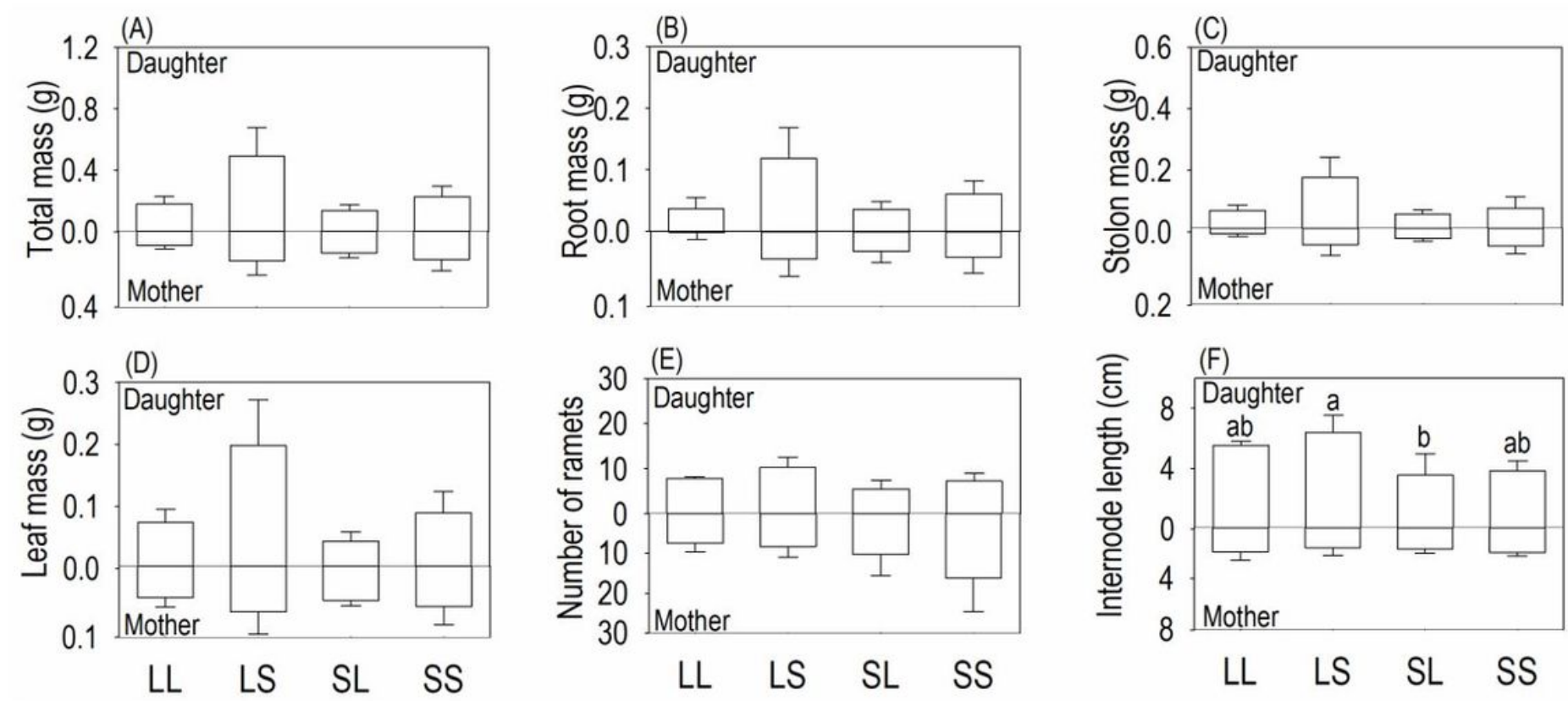

\section{Figure 3}

Total mass (A), root mass (B), stolon mass (C), leaf mass (D), number of ramets (E) and internode length (F) of the daughter (upper panel) and mother (lower panel) ramets of Glechoma longituba under the four treatments $(\mathrm{N}=4)$. Mean values $(\mathrm{SE})$ are given. Different letters indicate significant difference among the treatments. Conspecific-conditioned soil inoculation treatments: LL - "mother" and "daughter" ramets grown in soil inoculated with unsterilized conspecific soil, LS - mother with unsterilized and daughter with sterilised inoculum, SL - mother with sterilized and daughter with unsterilized inoculum, and SS - mother and daughter with sterilized inoculum. 